DrafT VERSION JUNE 2, 2018

Preprint typeset using $\mathrm{LT}_{\mathrm{E}} \mathrm{X}$ style emulateapj v. 08/22/09

\title{
ALMA OBSERVATIONS OF THE OUTFLOW FROM THE SOURCE I IN THE ORION-KL REGION
}

\author{
Luis A. Zapata ${ }^{1}$, Luis F. Rodríguez ${ }^{1}$, Johannes Schmid-BurgK ${ }^{2}$, Laurent Loinard $^{1,2}$, Karl M. Menten $^{2}$, and Salvador Curiel $^{3}$ \\ Draft version June 2, 2018
}

\begin{abstract}
In this Letter, we present sensitive millimeter $\mathrm{SiO}(\mathrm{J}=5-4 ; v=0)$ line observations of the outflow arising from the enigmatic object Orion Source I made with the Atacama Large Millimeter/Submillimeter Array (ALMA). The observations reveal that at scales of a few thousand AU, the outflow has a marked "butterfly" morphology along a northeast-southwest axis. However, contrary to what is found in the $\mathrm{SiO}$ and $\mathrm{H}_{2} \mathrm{O}$ maser observations at scales of tens of $\mathrm{AU}$, the blueshifted radial velocities of the moving gas are found to the northwest, while the redshifted velocities are in the southeast. The ALMA observations are complemented with $\mathrm{SiO}(\mathrm{J}=8-7 ; v=0)$ maps (with a similar spatial resolution) obtained with the Submillimeter Array (SMA). These observations also show a similar morphology and velocity structure in this outflow. We discuss some possibilities to explain these differences at small and large scales across the flow.
\end{abstract}

Subject headings: stars: pre-main sequence - ISM: jets and outflows - individual: (Orion Source I) - individual: (Orion $\mathrm{BN} / \mathrm{KL})$

\section{INTRODUCTION}

Since its discovery, the radio Source I in the middle of the Kleinmann-Low nebula in Orion, has always been an enigmatic object (Menten \& Reid 1995; Churchwell et al. 1987). Recent proper motion measurements suggest that the Orion Source I might have been part of a multiple system that disintegrated some 500 years ago (Gómez et al. 2008; Rodríguez et al. 2005; Goddi et al. 2011) originating an explosive outflow in the Orion $\mathrm{BN} / \mathrm{KL}$ region (Zapata et al. 2009; Bally et al. 2011; Zapata et al. 2011a). Bally et al. (2011) proposed that probably the Orion Source I is a shortperiod binary formed just before the stellar ejection and the formation of the explosive outflow. It is estimated that the separation of the binary is only a few Astronomical Units (AU) (Bally et al. 2011).

Strong $\mathrm{H}_{2} \mathrm{O}$ and $\mathrm{SiO}$ vibrationally excited $(v=1$ and 2) masers are concentrated very close to the Orion Source I. The $\mathrm{H}_{2} \mathrm{O}$ masers are distributed in an elongated pattern along a northeast-southwest axis and seem to be expanding about Source I (Genzel et al. 1981). The bulk of the $\mathrm{SiO}$ masers are found surrounding Source I and form an Xshaped structure; the emission in the southeast arms is predominantly blueshifted, while the emission in the northwest is predominantly redshifted. The intermediate-velocity emission is observed connecting the red and blue sides of the emission distribution. The $\mathrm{SiO}$ masers seem to be arising from a wide-angle bipolar wind emanating from a rotating, ionized edge-on disk with a southeast-northwest orientation (Matthews et al. 2010). The ionized disk has been imaged at $7 \mathrm{~mm}$. and appears to be surrounding a young massive star (Reid et al. 2007; Goddi et al. 2011).

At scales of a few hundreds of AU, Plambeck et al. (2009) imaged the outflow from the Orion Source I using CARMA (The Combined Array for Research in Millimeter-wave Astronomy) in the $\mathbf{J}=2-1$ from the vibrational ground state

\footnotetext{
${ }^{1}$ Centro de Radioastronomía y Astrofísica, UNAM, Apdo. Postal 3-72 (Xangari), 58089 Morelia, Michoacán, México

${ }^{2}$ Max-Planck-Institut für Radioastronomie, Auf dem Hügel 69, 53121, Bonn, Germany

${ }^{3}$ Instituto de Astronomía, Universidad Nacional Autónoma de México, Ap. 70-264, 04510 DF, México
}

of $\mathrm{SiO}$, and found that the central velocity channels showed the limb-brightened edges of two cones centered on Source I. However, since the outflow appears to lie nearly in the plane of the sky, there is no measurable red-blue velocity asymmetry in the cones, as observed for the masers.

We report $\sim 1.5^{\prime \prime}$ resolution images in the $\mathrm{SiO} \mathrm{J}=5-4 ; v=0$ obtained with the Atacama Large Millimeter/Submillimeter Array $^{4}$ (ALMA) from the Orion Source I. These observations are complemented with $\mathrm{SiO}(\mathrm{J}=8-7 ; v=0)$ maps obtained with the Submillimeter Array ${ }^{5}$ (SMA). Both observations reveal that the outflow from the Orion Source I has a "butterfly" morphology along a northeast-southwest axis. The blueshifted radial velocities are found to the northwest, while the redshifted velocities toward the southeast.

\section{OBSERVATIONS}

\subsection{ALMA}

The observations were made with sixteen antennas of ALMA on January 2012, during the ALMA science verification data program. The array at that point only included antennas with diameters of 12 meters. The 120 independent baselines ranged in projected length from 18 to $253 \mathrm{k} \lambda$. The phase reference center for the observations was at $\alpha_{J 2000.0}=$ $05^{\mathrm{h}} 35^{\mathrm{m}} 14^{\mathrm{s}} 35, \delta_{J 2000.0}=-05^{\circ} 22^{\prime} 35^{\prime \prime}$. 00 . The primary beam of ALMA at $217 \mathrm{GHz}$ has a FWHM 30". The emission from the outflow powered by the Orion Source I falls very well inside of the FWHM.

The ALMA digital correlator was configured in 4 spectral windows of $1875 \mathrm{MHz}$ and 3840 channels each. This provides a spectral resolution of $0.488 \mathrm{MHz}\left(\sim 0.7 \mathrm{~km} \mathrm{~s}^{-1}\right)$ per channel. As a special case, this observation consisted of 5 different spectral tunings resulting on 20 spectral windows. The spectral window where the line $\mathrm{SiO}(\mathrm{J}=5-4 ; v=0)$ at a

4 ALMA is a partnership of ESO (representing its member states), NSF (USA) and NINS (Japan), together with NRC (Canada) and NSC and ASIAA (Taiwan), in cooperation with the Republic of Chile. The Joint ALMA Observatory is operated by ESO, AUI/NRAO and NAOJ.

5 The Submillimeter Array (SMA) is a joint project between the Smithsonian Astrophysical Observatory and the Academia Sinica Institute of Astronomy and Astrophysics, and is funded by the Smithsonian Institution and the Academia Sinica. 


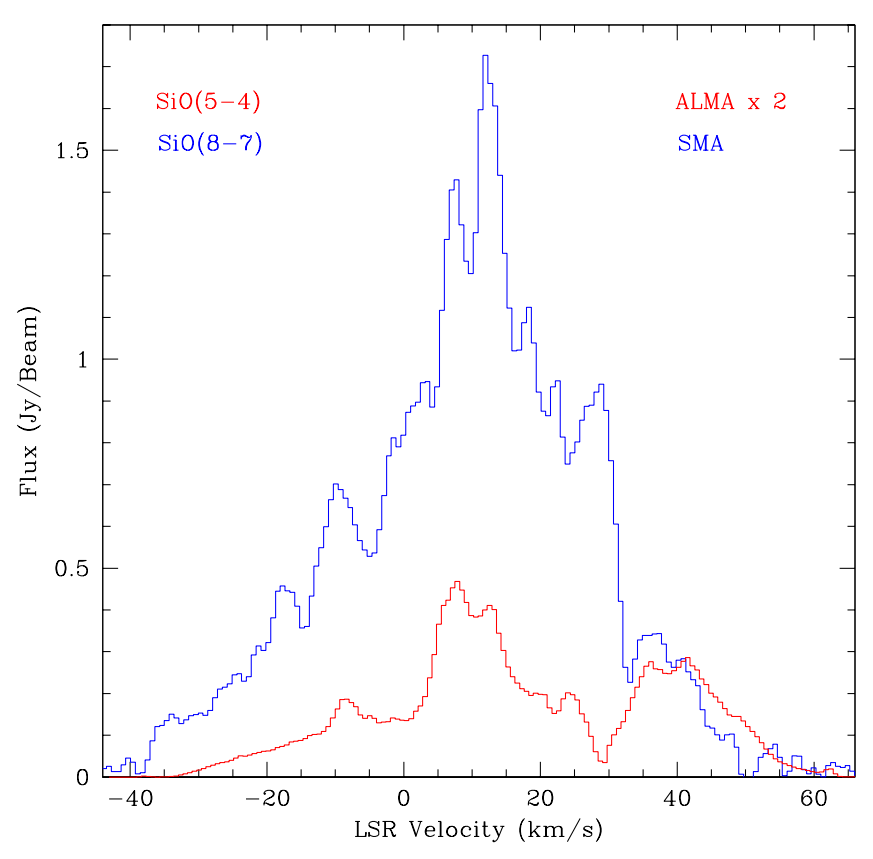

FIG. 1.- $\mathrm{SiO}(5-4)$ (red line) and $\mathrm{SiO}(8-7)$ (blue line) spectra from the Orion $\mathrm{BN} / \mathrm{KL}$ region obtained with the ALMA and SMA observations, respectively. The spectrum produced from the ALMA data is multiplied by a factor of two for convenience. In the ALMA data 1 Kelvin is equivalent to $9.04 \mathrm{Jy}$, while in the SMA data 1 Kelvin is equivalent to $1.73 \mathrm{Jy}$.

rest frequency of $217.10498 \mathrm{GHz}$ was detected, has a central frequency of $217.225 \mathrm{GHz}$.

Observations of Callisto provided the absolute scale for the flux density calibration while observations of the the quasar J0607-085 (with a flux of 1.4 Jy) provide the gain phase calibration. The total on-source integration time was about 100 min. This time was divided to observe the 5 spectral tunings. For time-dependent gain calibration, the nearby quasar J0607-085 was observed every 7 minutes.

The data were calibrated, imaged, and analyzed using the Common Astronomy Software Applications (CASA). To analyze the data, we also used the KARMA software (Gooch 1996). The resulting r.m.s. noise for the line images was about $100 \mathrm{mJy} \mathrm{beam}^{-1}$ at an angular resolution of 1 .' $^{\prime \prime} \times 18$.'23 with a P.A. $=-4.4^{\circ}$. We used uniform weighting to obtain a slightly better angular resolution.

\section{2. $S M A$}

The observations were obtained from the SMA, and were collected on December 2009, when the array was in its compact configuration. The 21 independent baselines in the compact configuration ranged in projected length from 16 to 70 $\mathrm{k} \lambda$. The phase reference center for the observations was at $\alpha_{J 2000.0}=05^{\mathrm{h}} 35^{\mathrm{m}} 15^{\mathrm{s}} 45, \delta_{J 2000.0}=-05^{\circ} 22^{\prime} 35^{\prime \prime}$. 0 . Two frequency bands, centered at $335.3306310 \mathrm{GHz}$ LSB (Lower Sideband) and $345.3306310 \mathrm{GHz}$ USB (Upper Sideband) were observed simultaneously. The observations were made in mosaicing mode using half-power point spacing between field centers and thus covering the entire $\mathrm{BN} / \mathrm{KL}$ region. The primary beam of the SMA at $345 \mathrm{GHz}$ has a FWHM $\sim 30^{\prime \prime}$.

The SMA digital correlator was configured in 24 spectral windows ("chunks") of $104 \mathrm{MHz}$ and 128 channels each. This provides a spectral resolution of $0.812 \mathrm{MHz}\left(\sim 0.7 \mathrm{~km} \mathrm{~s}^{-1}\right)$ per channel. The zenith opacity $\left(\tau_{230 G H z}\right)$, measured with the NRAO tipping radiometer located at the nearby Caltech Submillimeter Observatory, was about 0.07 , indicating excellent weather conditions during the observations. Observations of Uranus provided the absolute scale for the flux density calibration. The gain calibrators were the quasars J0541-056 and J0607-085, while 3C454.3 was used for bandpass calibration. The uncertainty in the flux scale is estimated to be between 15 and $20 \%$, based on the SMA monitoring of quasars. Further technical descriptions of the SMA and its calibration schemes can be found in Ho et al. (2004).

The data were calibrated using the IDL superset MIR, originally developed for the Owens Valley Radio Observatory (OVRO, Scoville et al. 1993) and adapted for the SMA. ${ }^{6}$ The calibrated data were imaged and analyzed in the standard manner using the MIRIAD and KARMA (Gooch 1996) softwares. We set the ROBUST parameter of the task INVERT to 0 to obtain an optimal compromise between resolution and sensitivity. The r.m.s. noise in each channel of the spectral line data was about $170 \mathrm{mJy}$ beam $^{-1}$ at an angular resolution of $2 .^{\prime \prime} 9 \times 1 .^{\prime \prime} 9$ with a P.A. $=+16.6^{\circ}$. The $\mathrm{SiO} \mathrm{J}=8-7 ; v=0$ was detected in the USB at a rest frequency of about $347.3 \mathrm{GHz}$.

\section{RESULTS}

In Figure 1, we show the total power $\mathrm{SiO}$ spectra of the ALMA and SMA observations toward the central part of the $\mathrm{BN} / \mathrm{KL}$ region. The total area from the spectra was obtained covers approximately one $\operatorname{arcmin}^{2}$ centered at the position of the Source I. No obvious maser emission is detected at these transitions. The spectra are very similar, but with that obtained at higher frequencies (SMA) being much stronger than the obtained with ALMA. This has also been observed in outflows associated with young low mass stars (e.g. HH 211; Lee et al. 2007; Palau et al. 2006) and is attributed to the $\mathrm{SiO}$ excitation within the outflow. The spectra have two central strong peaks, one peaking around of $+6 \mathrm{~km} \mathrm{~s}^{-1}$ and the second approximately at $+12 \mathrm{~km} \mathrm{~s}^{-1}$. These peaks are associated with the emission from the outflow of the Orion Source I around the systemic velocity of the cloud $\left(+9 \mathrm{~km} \mathrm{~s}^{-1}\right)$. The dip at $9 \mathrm{~km} \mathrm{~s}^{-1}$ could be self-absorption. The high-velocity structure has multiple components, some of them come from the outflow of the Orion Source I, but others come from the molecular bubble reported by Zapata et al. (2011b), and objects located in BN/KL. We estimated that the radial velocity of the Orion Source I is about $+6 \mathrm{~km} \mathrm{~s}^{-1}$, in good agreement with that value found by Plambeck et al. (2009) and Matthews et al. (2010).

The channel velocity map of the $\mathrm{SiO}(\mathrm{J}=5-4 ; v=0)$ emission from the Orion Source I mapped with ALMA is shown in Figure 2. The range of radial velocities $\left(-15.5\right.$ to $\left.+24.5 \mathrm{~km} \mathrm{~s}^{-1}\right)$ is in good agreement with the one obtained by Plambeck et al. (2009) shown in their Figure 1. However, our maps with lower angular resolution seem to recover some extended parts of the outflow lost in their maps. This is more evident in our channels maps with central velocities of $-15.5 \mathrm{~km} \mathrm{~s}^{-1}$ and $+14.5 \mathrm{~km} \mathrm{~s}^{-1}$. In these channels, we observe some northsouth structures with a "wing" shape, extending in an eastwest orientation. The emission arising from the central velocity channels could not be resolved with our actual resolution, as in Plambeck et al. (2009).

Integrated intensity maps (moment 0 ) of the $\mathrm{SiO}$ emission

6 The MIR-IDL cookbook by C. Qi can be found at http://cfa-www.harvard.edu/ cqi/mircook.html 


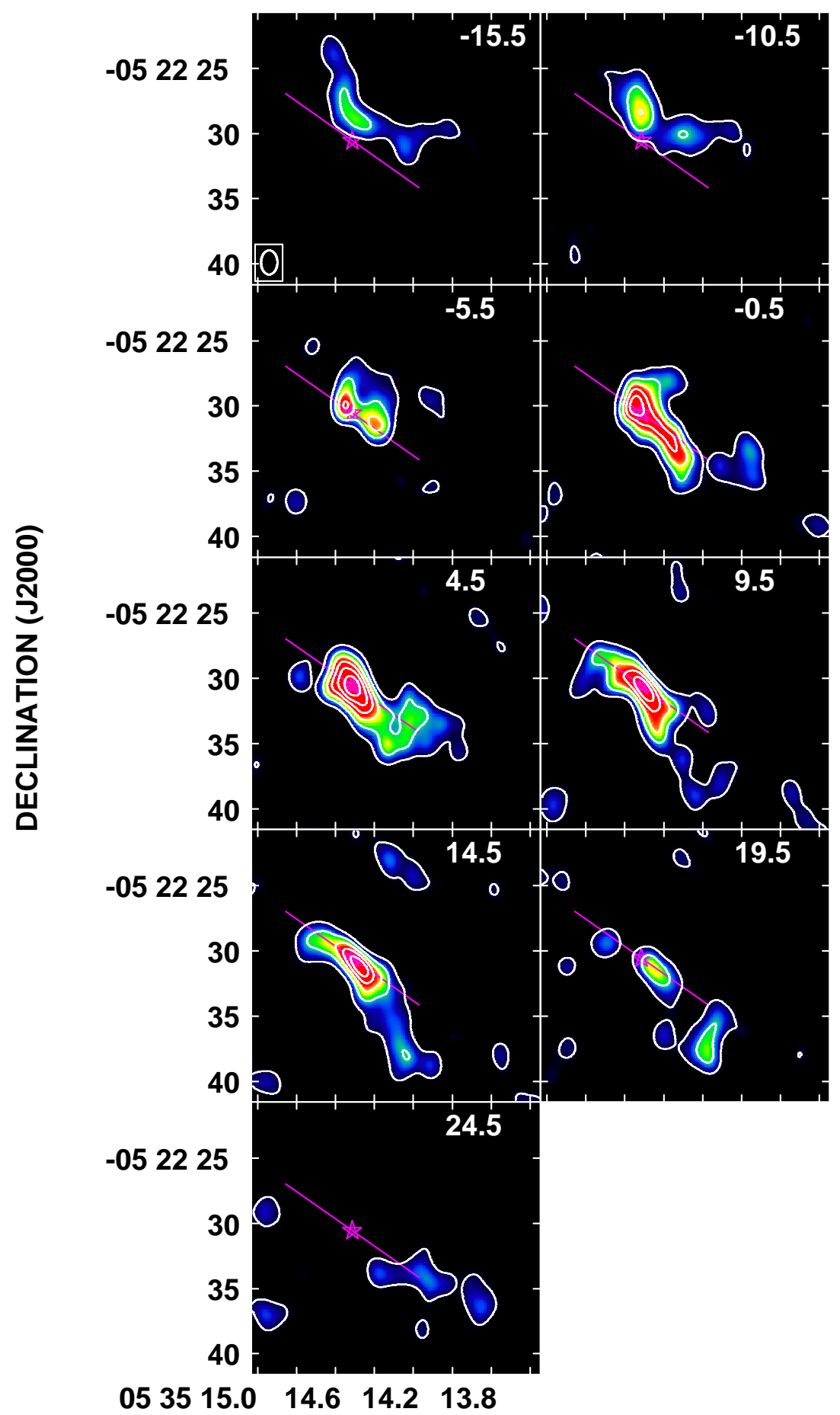

RIGHT ASCENSION (J2000)

FIG. 2. - ALMA channel map of the $\mathrm{SiO}(\mathrm{J}=5-4 ; v=0)$ line toward the Orion Source I. Every velocity channel is $5.0 \mathrm{~km} \mathrm{~s}^{-1}$ wide and is centered close to the position of the Orion Source I. Each box is labeled with the central LSR velocity in $\mathrm{km} \mathrm{s}^{-1}$. A pink star marks the location of radio Orion Source I. The line represents the orientation of the outflow at a P.A. $=55^{\circ}$ Plambeck et al.2009). The contours are from $-5,5,10,15,20,25,30,35,40,45,50,55$, and 60 times 0.14 Jy beam $^{-1}$, the rms noise of the image. The half-power contour of the synthesized beam is shown in the bottom left corner of the first panel. 


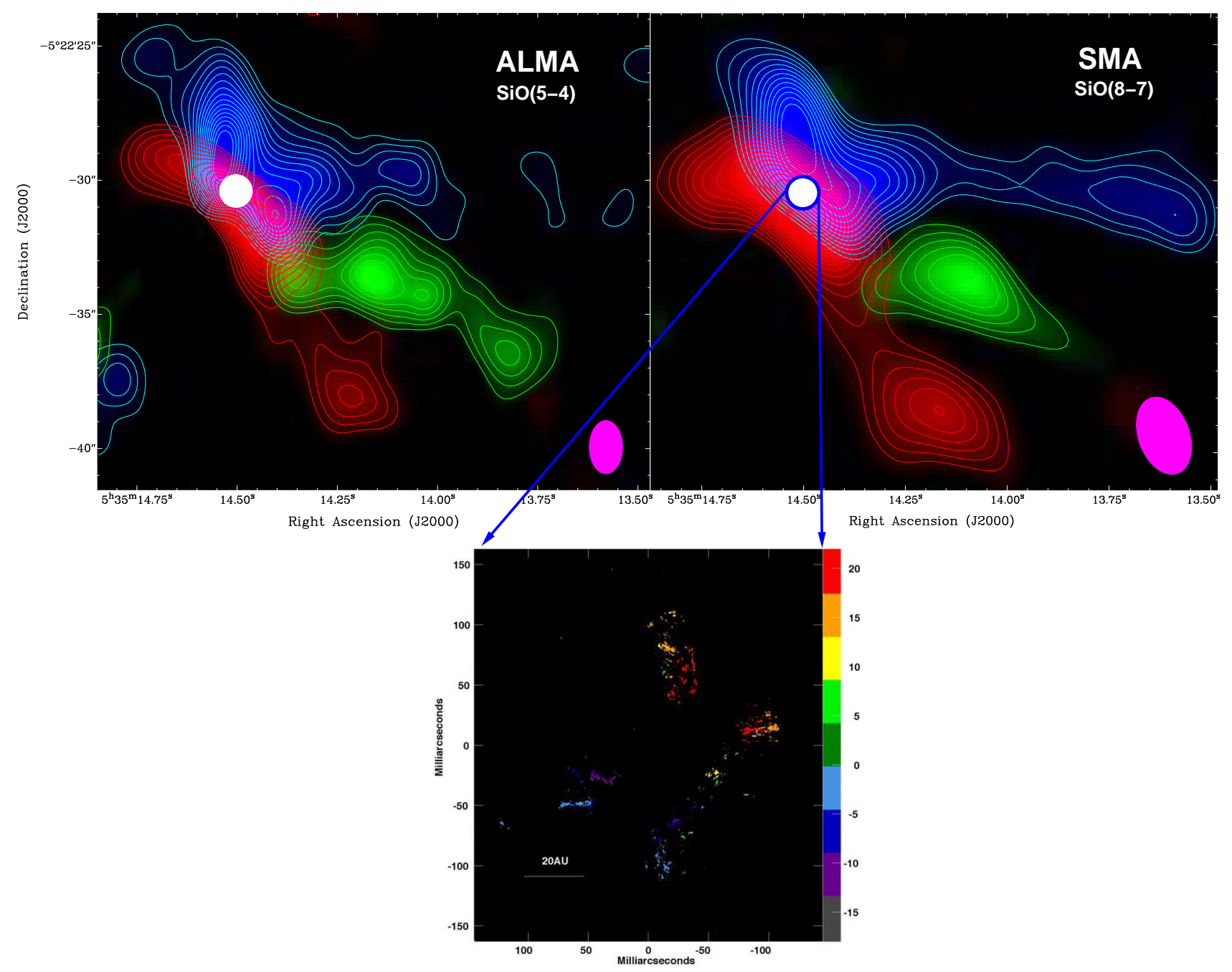

FIG. 3.- Upper panels: ALMA (left) and SMA (right) integrated intensity (moment 0) color-scale and contour maps of the SiO thermal emission from the outflow arising from the Orion Source I. The blue color-scale and contours represent blueshifted gas with LSR velocities ranking from $-15 \mathrm{~km} \mathrm{~s}^{-1}$ to $-5 \mathrm{~km} \mathrm{~s}{ }^{-1}$. The red color-scale and contours represent redshifted gas with LSR velocities ranging from $+15 \mathrm{~km} \mathrm{~s}^{-1}$ to $+25 \mathrm{~km} \mathrm{~s}^{-1}$. The green color-scale and contours represent redshifted high velocity gas with LSR velocities ranging from $+26 \mathrm{~km} \mathrm{~s}^{-1}$ to $+30 \mathrm{~km} \mathrm{~s}^{-1}$. In the left panel, the blue and red contours range from $-10 \%$ to $90 \%$ of the peak emission, in steps of $5 \%$. The emission peaks for the blueshifted and redshifted emission are 28 and $59 \mathrm{Jy} \mathrm{beam}^{-1} \mathrm{~km} \mathrm{~s}^{-1}$. The green contours range from $-25 \%$ to $90 \%$ of the peak emission, in steps of $10 \%$. The emission peak for the redshifted emission (associated with the green color) is $27 \mathrm{Jy} \mathrm{beam}^{-1} \mathrm{~km} \mathrm{~s}^{-1}$. In the right panel, the blue and red contours range from $-20 \%$ to $90 \%$ of the peak emission, in steps of $5 \%$. The emission peaks for the blueshifted and redshifted emission are 140 and $350 \mathrm{Jy} \mathrm{beam}^{-1} \mathrm{~km} \mathrm{~s}^{-1}$, respectively. The green contours range from $40 \%$ to $90 \%$ of the peak emission, in steps of $10 \%$. The emission peak for the redshifted emission (associated with the green color) is $100 \mathrm{Jy} \mathrm{beam}^{-1} \mathrm{~km} \mathrm{~s}^{-1}$. In both panels, the synthesized beams of the observations are shown in the lower right corner. The white dot marks the position of the Orion Source I, as derived from VLA proper-motion data (Gómez et al. 2008). Lower panel: This image was obtained from Matthews et al. (2010) and displays the velocity field of the ${ }^{28} \mathrm{SiO} v=1$ and $v=2, \mathrm{~J}=1-0$ emission surrounding the Orion Source $\mathrm{I}$.

obtained with ALMA and SMA are presented in Figure 3. In these maps, we only integrated over redshifted and blueshifted radial velocities associated with the outflow from the Orion Source I. The blueshifted gas emission was integrated over LSR velocities ranging from $-15 \mathrm{~km} \mathrm{~s}^{-1}$ to $-5 \mathrm{~km} \mathrm{~s}^{-1}$. The redshifted gas emission was integrated over the LSR velocities range from $+15 \mathrm{~km} \mathrm{~s}^{-1}$ to $+25 \mathrm{~km} \mathrm{~s}^{-1}$. The very high redshifted velocities range from $+26 \mathrm{~km} \mathrm{~s}^{-1}$ to $+30 \mathrm{~km} \mathrm{~s}^{-1}$. These maps reveal that the outflow from the Orion Source I has a marked "butterfly" morphology along a northeastsouthwest axis, with the blueshifted radial velocities found to the northwest, while the redshifted velocities are toward the southeast. This velocity structure is different of what is observed at smaller scales, see the Figure 7 of Matthews et al. (2010) or our Figure 3. The ranges of radial velocities of the $\mathrm{SiO}$ masers are similar to those obtained by us. The wings of the "butterfly" shape extend several arcseconds along the east-west direction.

At the middle of the "wings", we find very high velocity emission (see Figure 2, the green color-scale and contours) that also appears to emanate from the Source I, this component is observed at small scales with the same orientation by Matthews et al. (2010). In the ALMA map, with a better sensitivity and angular resolution than the SMA one, the tail extends a few more arcseconds. The "butterfly" morphology can also be seen in the $0.5^{\prime \prime}$ resolution map of Wright et al. (1995) in the $\mathrm{SiO}(\mathrm{J}=2-1 ; v=0)$ and in Figure 3 of Plambeck et al. (2009) which was derived from 1 ' resolution data. The wings of the butterfly also show some differences between both maps. The red wing is only half, different from the blue wing that is shaped like an U in the ALMA map. Something seems to suppress the other red half. Maybe it is due to some density inhomogeneities in the surroundings of the bipolar outflow.

\section{DISCUSION}




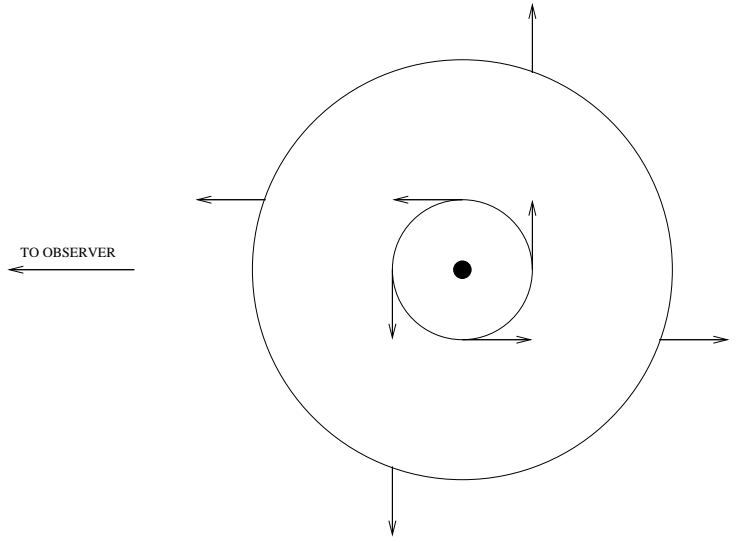

FIG. 4.- The central dot marks the position of the star and the inner circle indicates a rotating disk. Gas ejected ballistically from the disk will appear, at larger scales, radially expanding (and not rotating).

In the following, we will discuss some possibilities trying to explain the nature of the velocity structure and morphology of the outflow emanating from Source I.

\subsection{Two bipolar outflows}

A likely possibility is to have two bipolar outflows emanating from a short-period binary star located in the middle of the ionized disk of the Source I. This is in agreement with what was proposed by Bally et al. (2011) about the binarity of the Source I. One outflow could be moving in a nearly northsouth orientation with its blueshifted side in the north and the redshifted side toward the south. The second bipolar outflow, would have an east-west axis, with its blueshifted side to the west, while its redshifted side is to the east. The difference in size of the components could be attributed to the molecular cloud inhomogeneities. The fact that a binary could have a strong precession moving in short time scales, might explain the difference in the redshifted and blueshifted velocities observed at different scales. The $\mathrm{SiO}$ masers could be tracing the innermost parts of the two bipolar outflows ejected at the present time (Matthews et al. 2010). However, this hypothesis could not explain satisfactorily well the high velocity tail (with a southwest orientation) observed in the middle of the outflows, and the observations of the single "cone" outflow found by Plambeck et al. (2009) at their central channel velocities.

\subsection{Ballistic ejecta from a rotating disk}

We also consider the possibility that the thermal $\mathrm{SiO}$ traces gas that was ejected ballistically in the past from the smallscale disk associated with Source I. However, Figure 4 shows that the gas ejected ballistically at small scales from a rotating structure will, at large scales, appears as an expanding structure, with the largest radial velocities overlapping at the center of the structure. This is not what is observed and we rule out this possibility.

\subsection{A single expanding wide-angle NE-SW outflow}

Another possibility is having a wide-angle expanding outflow that is emanating from the ionized, nearly edge-on, rotating disk reported by Matthews et al. (2010) and Reid et al. (2007). In this case, the $\mathrm{SiO}$ masers would trace the innermost parts of the outflow and the thermal $\mathrm{SiO}$ emission would be tracing the outer parts of the outflow. The difference in the orientation of the radial velocities at different scales could be attributed to that maybe in the past the rotation of the disk was in a different sense. This could have happened if a large amount of matter fell directly to the disk and changed the rotation sense. This third idea might explain most of the characteristics observed in Figure 3 and those observed at small scales by Matthews et al. (2010) and Plambeck et al. (2009). However, the idea of a disk inverting its rotation direction requires of unusual events during the evolution of the disk and deserves further theoretical and observational studies.

\section{CONCLUSIONS}

In this paper, we have reported $\mathrm{SiO}(\mathrm{J}=5-4 ; \nu=0)$ and $\mathrm{SiO}(\mathrm{J}=8-7 ; v=0)$ observations obtained with ALMA and the SMA. We found a marked "butterfly" morphology along a northeast-southwest axis in the outflow from the Source I. However, the blueshifted radial velocities are found to the northwest, while the redshifted velocities toward the southeast, which is contrary to what is observed at very small scales. We discussed some possibilities to explain this phenomenon, however, any of these hypotheses requires further sensitive, and high spatial resolution observations.

This paper makes use of the following ALMA Science Verification data: ADS/JAO.ALMA\#2011.0.00009.SV L.A.Z, L. L. and L. F. R. acknowledge the financial support from DGAPA, UNAM, and CONACyT, México. L. L. is indebted to the Alexander von Humboldt Stiftung for financial support. We are very thankful for the suggestions of anonymous referee that helps to improve our manuscript.

\section{REFERENCES}

Bally, J., Cunningham, N. J., Moeckel, N., et al. 2011, ApJ, 727, 113

Churchwell, E., Felli, M., Wood, D. O. S., \& Massi, M. 1987, ApJ, 321, 516

Ho, P. T. P., Moran, J. M., \& Lo, K. Y. 2004, ApJ, 616, L1

Genzel, R., Reid, M. J., Moran, J. M., \& Downes, D. 1981, ApJ, 244, 884

Goddi, C., Humphreys, E. M. L., Greenhill, L. J., Chandler, C. J., \&

Matthews, L. D. 2011, ApJ, 728, 15

Gooch, R. 1996, Astronomical Data Analysis Software and Systems V, 101, 80

Gómez, L., Rodríguez, L. F., Loinard, L., et al. 2008, ApJ, 685, 333

Lee, C.-F., Ho, P. T. P., Palau, A., et al. 2007, ApJ, 670, 1188

Matthews, L. D., Greenhill, L. J., Goddi, C., et al. 2010, ApJ, 708, 80

Menten, K. M., \& Reid, M. J. 1995, ApJ, 445, L157

Palau, A., Ho, P. T. P., Zhang, Q., et al. 2006, ApJ, 636, L137

Plambeck, R. L., Wright, M. C. H., Friedel, D. N., et al. 2009, ApJ, 704, L25
Scoville, N. Z., Carlstrom, J. E., Chandler, C. J., et al. 1993, PASP, 105, 1482

Reid, M. J., Menten, K. M., Greenhill, L. J., \& Chandler, C. J. 2007, ApJ, 664,950

Rodríguez, L. F., Poveda, A., Lizano, S., \& Allen, C. 2005, ApJ, 627, L65 Wright, M. C. H., Plambeck, R. L., Mundy, L. G., \& Looney, L. W. 1995, ApJ, 455, L185

Zapata, L. A., Schmid-Burgk, J., Ho, P. T. P., Rodríguez, L. F., \& Menten, K. M. 2009, ApJ, 704, L45

Zapata, L. A., Schmid-Burgk, J., \& Menten, K. M. 2011b, A\&A, 529, A24

Zapata, L. A., Loinard, L., Schmid-Burgk, J., et al. 2011a, ApJ, 726, L12 